\title{
Pregnancy outcomes in bone marrow disorders: a tertiary care experience
}

\author{
Rao Preethi Venkatachala, C N Sheela
}

\author{
Corresponding author: Dr. Rao Preethi Venkatachala, Assistant Professor, Department of \\ Obstetrics and Gynaecology, St Johns Medical College and Hospital, Bengaluru, Karnataka, India; \\ Email : preethibhat77@gmail.com
}

Distributed under Attribution-Non Commercial - Share Alike 4.0 International (CC BY-NC-SA 4.0)

\begin{abstract}
Objectives: To study the maternal and foetal outcomes in pregnancies complicated by bone marrow disorders. Material and methods: A retrospective descriptive study of women with bone marrow disorders in pregnancy, between January 2011 and January 2016 was carried out. Their antenatal and labour records were reviewed and demographic details noted. The primary outcome measures studied were - severity of anaemia, additional drugs required during pregnancy, gestational age at delivery, requirement of transfusion of blood and blood products and mode of delivery. Obstetric and medical complications and neonatal outcomes were the secondary outcome measures noted. Blood counts during pregnancy and after delivery were noted. Data are presented as descriptive statistics including means and percentage. Results: Prevalence of bone marrow disorder complicating pregnancy was (10/12420) $0.08 \%$. 6 women had aplastic anaemia, 2 had CML, 1 had AML and 1 had myelodysplastic syndrome. $50 \%$ were in remission; $60 \%$ received drugs for bone marrow disorder. Antenatal complications like preeclampsia (40\%), oligohydramnios (40\%), infections, IUGR and IUD (30\% each), preterm labour, previous caesarean delivery (20\% each) and PNH (10\%) were noted. 50\% had moderate anaemia, 30\% severe anaemia, $90 \%$ had thrombocytopenia. Transfusion of blood products was required in 30\% antepartum, 70\% intrapartum and $60 \%$ postpartum. 50\% had vaginal delivery. There was no PPH or maternal mortality. $70 \%$ took home healthy babies. Conclusion: Multidisciplinary approach, preconceptional counseling, good antenatal care, delivery at a tertiary care centre, prompt treatment of infections and sepsis, prophylactic transfusions and optimal use of chemotherapeutic drugs can ensure successful pregnancy.
\end{abstract}

Keywords: Bone marrow disorder, aplastic anaemia, acute myeloid leukaemia, chronic myeloid leukaemia, myelodysplastic syndromes, pregnancy outcomes.

Pregnancy complicated by bone marrow disorders, though rare, is a serious condition. Marrow damage and dysfunction may be due to deficient haematopoeisis or secondary to infection, inflammation or cancer ${ }^{1}$. Many of these disorders share an immune mediated mechanism of marrow destruction and some element of genomic instability resulting in higher rates of malignant transformation ${ }^{1}$.

Bone marrow disorders are characterized by various degrees of cytopenias and include a spectrum of disorders varying from marrow hypocellularity as in aplastic anaemia (AA) to myelodysplasia (MDS), pure red cell aplasia and myelophthistic conditions ${ }^{1}$. Acute or chronic myeloid leukaemias (AML, CML) are clonal hematopoetic stem cell disorders ${ }^{1}$. They lead to loss of normal marrow function, diminishing the production of normal red cells, white cells and platelets.

Maternal complications include haemorrhage, sepsis, abortions, preterm labour and birth, oligohydramnios, preeclampsia, gestational diabetes and cardiac failure while foetus may suffer from intrauterine growth restriction (IUGR) and even intra uterine death (IUD), low birth weight (LBW) and neonatal asphyxia ${ }^{2-6}$.

Received: $2^{\text {st }}$ July 2020, Peer review completed: $20^{\text {th }}$ October 2020, Accepted: $5^{\text {th }}$ January 2021.

Venkatachala RP, Sheela CN. Pregnancy outcomes in bone marrow disorders: a tertiary care experience. The New Indian Journal of OBGYN. 2021; 8(1): 81-8. 
Termination of pregnancy produces favourable effect in terms of remission and survival, if detected in early gestation, especially when there is severe aplasia $2,3,6,7$. Immunosuppressive therapy and bone marrow transplantation are contraindicated in pregnancy due to potential toxicity to foetus ${ }^{2,3,8}$. Haemopoetic growth factors cannot be recommended unless more reports on its use in non pregnant patients become available as their efficacy is also equivocal $^{4,6}$.

These bone marrow failure states can cause increase in maternal and perinatal morbidity and mortality, but there is scant literature describing them. Hence we have undertaken this study to review the pregnancy outcomes in women with bone marrow disorders.

\section{Materials and methods}

A retrospective descriptive study of women with bone marrow disorders in pregnancy, who delivered at SJMCH between January 2011 and January 2016, was carried out. Women with nutritional anaemias (iron deficiency, megaloblastic anaemia), anaemia due to acute or chronic blood loss and hemolytic anaemias were excluded from the study.

Antenatal and labour records were reviewed. Demographic variables like maternal age, parity, booking status, type of bone marrow disorder, duration of the disease, drug intake 3 months prior to or at first visit, disease status at the beginning of pregnancy and presenting complaints were noted. The primary outcome measures studied were severity of anaemia defined as per Indian Council for Medical Research (ICMR) criteria (Hb $10-10.9 \mathrm{~g} / \mathrm{dl}$ as mild; $\mathrm{Hb} 9.9$ - $7 \mathrm{~g} / \mathrm{dl}$ as moderate; $\mathrm{Hb} 6.9$ - $4 \mathrm{~g} / \mathrm{dl}$ as severe and $\mathrm{Hb}<4 \mathrm{~g} / \mathrm{dl}$ as very severe anaemia) ${ }^{9}$, additional drugs/treatment required during pregnancy, gestational age at delivery, requirement of transfusion of blood and blood products and mode of delivery. Obstetric and medical complications and neonatal outcomes such as birth asphyxia, need for NICU care, congenital anomalies were the secondary outcome measures noted. Blood counts [haemoglobin $(\mathrm{Hb})$, total leucocyte count (TC), neutrophil count, platelet count, reticulocyte count, prothrombin time (PT), INR, activated partial thromboplastin time (APTT)] of pregnant women during pregnancy and after delivery, at discharge were also noted. Where several measurements were available for a particular period, the lowest value was chosen. Data are presented as descriptive statistics including means and percentage.
The severity of AA was categorized as per established criteria ${ }^{10,11}$. Complete remission of AA was defined as hemoglobin concentration of $12 \mathrm{~g} / \mathrm{dL}$, a neutrophil count of $1.5 \times 10^{9}$ cells/L or greater, platelet count of $150 \times 10^{9}$ cells/L or greater, and no need for transfusion. Patients who did not fulfill the haematologic criteria for complete remission but did not require transfusion and had no evidence of paroxysmal nocturnal hemoglobinuria (PNH) or MDS were considered to have partial remission. Relapse was defined as a decrease in blood counts to a platelet count less than $20 \times 10^{9}$ cells/L or to counts necessitating regular packed cell or platelet transfusions.

The study was approved by the Institutional Ethics Committee.

\section{Results}

The prevalence of bone marrow disorders complicating pregnancy was $10 / 12420(0.08 \%)$. Among the 10 women, 6 had aplastic anaemia (2 of whom had very severe AA), 2 had CML, 1 had AML - M2 and 1 had steroid resistant myelodysplastic syndrome (MDS). The demographic details are shown in table 1. The complications, treatment, maternal and foetal outcomes are presented in table 2. The hematological profile of the patients during pregnancy and after delivery is shown in table 3.

The mean maternal age was 26.3 years $(21-30$ y); there were 4 primi and 6 multigravidae; $5(50 \%)$ women were booked. The bone marrow disorder was detected for the first time during pregnancy in $3(30 \%)$ women. In the others, the duration of bone marrow failure varied from 3 to 11 years. $60 \%$ had received drugs for the same, 3 months prior to pregnancy or at first visit. $50 \%$ were in remission. $40 \%$ presented with hypertension, 30\% with IUD and 20\% each with preterm labour and anaemia at time of admission. Antenatal complications like preeclampsia and oligohydramnios were noted in $40 \%$ each, infections, IUGR, and IUD in $30 \%$ each, preterm labour, placenta praevia, previous caesarean delivery and diabetes mellitus in $20 \%$ each and $\mathrm{PNH}$ in 10\%. 50\% had moderate anaemia, 30\% severe anaemia and $20 \%$ did not have anaemia during pregnancy. $30 \%$ required antenatal transfusions of blood products.

The mean gestational age at delivery was 36 weeks. Of the 10 women, 5(50\%) had vaginal deliveries and $5(50 \%)$ underwent caesarean deliveries for obstetric indications. Prophylactic transfusion of packed cells or platelet concentrates was given at the time of induction of labour or anaesthesia and at delivery in $70 \%$ of women. Postpartum 


\begin{tabular}{|c|c|c|c|c|c|c|c|c|}
\hline Case & $\begin{array}{l}\text { Age in } \\
\text { year }\end{array}$ & Parity & $\begin{array}{l}\text { Booking } \\
\text { status }\end{array}$ & $\begin{array}{l}\text { Type of bone } \\
\text { marrow disorder }\end{array}$ & $\begin{array}{l}\text { Duration } \\
\text { of disease }\end{array}$ & $\begin{array}{l}\text { Drug intake for } \\
\text { bone marrow } \\
\text { disorder }\end{array}$ & $\begin{array}{l}\text { Pre pregnancy } \\
\text { disease status }\end{array}$ & $\begin{array}{l}\text { Presenting } \\
\text { complaint }\end{array}$ \\
\hline Mrs M & 25 & Primi & Unbooked & Aplastic anaemia & $\begin{array}{l}\text { Current } \\
\text { pregnancy } \\
\text { at } 34 \text { weeks }\end{array}$ & Nil & $\begin{array}{l}\text { Diagnosed in } \\
\text { this pregnancy }\end{array}$ & $\begin{array}{l}\text { Dyspnoea on } \\
\text { exertion, } \\
\text { Fever, Preterm } \\
\text { labour }\end{array}$ \\
\hline Mrs M & 28 & G2P1L1 & Unbooked & Aplastic anaemia & 3 years & Nil & Remission & $\begin{array}{l}\text { Preterm labour } \\
\text { Increased BP }\end{array}$ \\
\hline Mrs S & 30 & Primi & Unbooked & $\begin{array}{l}\text { Aplastic anaemia } \\
\text { with PNH }\end{array}$ & 11 years & $\begin{array}{l}\text { Danazol prior to } \\
\text { pregnancy }\end{array}$ & Relapse & $\begin{array}{l}\text { IUD } \\
\text { Hypokalemia }\end{array}$ \\
\hline Mrs B & 21 & Primi & Unbooked & Aplastic anaemia & 3 years & $\begin{array}{l}\text { Conceived while } \\
\text { on Methyl } \\
\text { prednisolone, } \\
\text { stopped at } 2^{\text {nd }} \\
\text { month }\end{array}$ & Remission & $\begin{array}{l}\text { IUD } \\
\text { Increased BP }\end{array}$ \\
\hline Mrs B & 24 & G2P1 & Booked & Aplastic anaemia & 6 years & Nil & Remission & Preterm labour \\
\hline Mrs M & 28 & G2P1 & Booked & Aplastic anaemia & 3 years & $\begin{array}{l}\text { Cyclosporine and } \\
\text { Danazol prior to } \\
\text { pregnancy, } \\
\text { stopped } 3 \text { months } \\
\text { prior to } \\
\text { conception }\end{array}$ & Remission & Nil \\
\hline Mrs S & 21 & Primi & Booked & $\begin{array}{l}\text { Chronic myeloid } \\
\text { leukaemia }\end{array}$ & $\begin{array}{l}\text { Current } \\
\text { pregnancy } \\
\text { at } 5 \text { weeks }\end{array}$ & $\begin{array}{l}\text { Imatinib since } 7 \mathrm{w} \\
\text { gestation }\end{array}$ & $\begin{array}{l}\text { Diagnosed in } \\
\text { this pregnancy }\end{array}$ & IUD \\
\hline Mrs BR & 27 & G2A1 & Booked & $\begin{array}{l}\text { Chronic myeloid } \\
\text { leukaemia }\end{array}$ & 3 years & $\begin{array}{l}\text { Imatinib prior to } \\
\text { pregnancy, } \\
\text { stopped after } \\
\text { conception, } \\
\text { restarted at } 24 \mathrm{w} \\
\text { due to blast crisis }\end{array}$ & Remission & Nil \\
\hline Mrs MB & 30 & G3P2L2 & Unbooked & $\begin{array}{l}\text { Acute myeloid } \\
\text { leukaemia M2 }\end{array}$ & $\begin{array}{l}\text { Current } \\
\text { pregnancy } \\
\text { at } 37 \text { weeks }\end{array}$ & Nil & $\begin{array}{l}\text { Diagnosed in } \\
\text { this pregnancy }\end{array}$ & Increased BP \\
\hline Mrs S & 29 & G3P1L1A1 & Booked & $\begin{array}{l}\text { Myelodysplastic } \\
\text { syndrome }\end{array}$ & 8 years & $\begin{array}{l}\text { Cyclosporine } \\
\text { prior to } \\
\text { pregnancy, } \\
\text { changed to } \\
\text { Danazol which } \\
\text { was continued till } \\
18 \text { wks }\end{array}$ & Relapse & $\begin{array}{l}\text { Shortness of } \\
\text { breath tiredness, } \\
\text { Increased BP }\end{array}$ \\
\hline
\end{tabular}

transfusions were required in $60 \%$. There was no postpartum haemorrhage (PPH) in any of these women. Fever (30\%) and puerperal sepsis $(20 \%)$ were among the postpartum complications observed. 3 (30\%) women required intensive care (ICU care) - 1 with AA for suspected peripartum cardiomyopathy, 1 with AML for pneumonia and 1 with MDS and chronic hypertension for worsening maternal condition.

There were 3 intra uterine demises - all in $3^{\text {rd }}$ trimester. The mean birth weight of babies was $2.06 \mathrm{~kg}$ (1.5-3.4 kg). All the live babies were healthy with good Apgar scores. 3 babies required NICU care for preterm birth or low birth weight. 1 woman with AA had a congenital anomaly (pleuro amnion shunt) leading to non immune hydrops, polyhydramnios and intra uterine demise.
$90 \%$ women had thrombocytopenia. 3 with aplastic anaemia had leucopenia, 1 with CML and 1 with AML had leucocytosis. After delivery, at discharge, 2 women did not have anaemia, 1 had mild, 5 had moderate and 1 had severe anaemia while 4 had thrombocytopenia. Neutrophil counts and TC improved after delivery in all patients.

Among the 6 women with AA, 2 had very severe disease; $5(83 \%)$ had a prior diagnosis of AA; 3(50\%) had oligohydramnios and preterm labour, 2(33\%) had preeclampsia, IUGR, IUD, diabetes, placenta praevia and $1(16 \%)$ had multiple pregnancy and infection each. 4(66\%) had moderate anaemia and $1(16 \%)$ had severe anaemia. $4(66 \%)$ had vaginal deliveries and $2(33 \%)$ caesarean delivery. $4(66 \%)$ received transfusions intra and postpartum. 


\begin{tabular}{|c|c|c|c|c|c|c|c|c|}
\hline \multirow[t]{2}{*}{ Case } & \multirow{2}{*}{$\begin{array}{l}\text { Antenatal } \\
\text { complications }\end{array}$} & \multirow{2}{*}{$\begin{array}{l}\text { Additional } \\
\text { drugs }\end{array}$} & \multirow{2}{*}{$\begin{array}{l}\text { Gestatio- } \\
\text { nal age at } \\
\text { delivery in } \\
\text { weeks }\end{array}$} & \multirow{2}{*}{$\begin{array}{l}\text { Mode of } \\
\text { delivery }\end{array}$} & \multicolumn{2}{|c|}{ Blood products transfused } & \multirow{2}{*}{$\begin{array}{l}\text { Post- } \\
\text { partum } \\
\text { complic- } \\
\text { ations }\end{array}$} & \multirow{2}{*}{$\begin{array}{l}\text { Neonatal } \\
\text { outcome }\end{array}$} \\
\hline & & & & & Intra-partum & $\begin{array}{l}\text { Post- } \\
\text { partum }\end{array}$ & & \\
\hline $\begin{array}{l}\text { Mrs } \\
M\end{array}$ & $\begin{array}{l}\text { Moderate anaemia, } \\
\text { fever, anhydramnios, } \\
\text { breech, type } 2 \\
\text { diabetes mellitus }\end{array}$ & Nil & $35^{+5}$ & $\begin{array}{l}\text { Emergency } \\
\text { LSCS } \\
\text { Indication- } \\
\text { breech, anhydra- } \\
\text { mnios }\end{array}$ & Nil & $\begin{array}{l}1 \text { unit } \\
\text { Packed } \\
\text { cells on } \\
\text { POD } 7 \& 8\end{array}$ & Fever & $\begin{array}{l}\text { Healthy, } \\
\text { girl, } 1.6 \mathrm{~kg}\end{array}$ \\
\hline $\begin{array}{l}\text { Mrs } \\
M\end{array}$ & $\begin{array}{l}\text { Moderate anaemia } \\
\text { Infections UTI, } \\
\text { Candidiasis, } \\
\text { Typhoid, Type } 2 \\
\text { Diabetes mellitus, } \\
\text { DCDA Twins, } \\
\text { Preeclampsia, } \\
\text { Oligohydramnios } \\
\text { Previous LSCS, } \\
\text { Preterm labour }\end{array}$ & Nil & $35^{+6}$ & $\begin{array}{l}\text { Emergency } \\
\text { LSCS } \\
\text { Indn- DCDA } \\
\text { Twins, } \\
\text { Preeclampsia } \\
\text { Oligohydra- } \\
\text { mnios } \\
\text { Previous LSCS } \\
\text { Preterm labour }\end{array}$ & $\begin{array}{l}1 \text { unit Packed } \\
\text { cells, } \\
1 \text { unit } \\
\text { Pheresced } \\
\text { platelets }\end{array}$ & $\begin{array}{l}\text { units } \\
\text { random } \\
\text { donor } \\
\text { platelets on } \\
\text { POD3 }\end{array}$ & $\begin{array}{l}\text { Puerperal } \\
\text { sepsis - UTI, } \\
\text { Fever } \\
\text { ICU care for } \\
\text { peripartum } \\
\text { cardio- } \\
\text { myopathy }\end{array}$ & $\begin{array}{l}\text { Healthy, } \\
\text { Twin 1-girl, } \\
1.54 \mathrm{~kg}, \\
\text { Twin 2- } \\
\text { girl, } \\
1.51 \mathrm{~kg}\end{array}$ \\
\hline Mrs S & $\begin{array}{l}\text { Severe anaemia at } 18 \\
\text { weeks, PNH at } 36 \\
\text { weeks, Placenta } \\
\text { praevia, } \\
\text { Polyhydramnios, } \\
\text { Non immumne } \\
\text { hydrops foetalis, IUD }\end{array}$ & $\begin{array}{l}\text { Inj Vitamin } \\
\text { B12 at } 18 \\
\text { wks, } 4 \text { units } \\
\text { Platelets, } \\
1 \text { unit Packed } \\
\text { cells at } 36 \text { wks } \\
\text { for PNH }\end{array}$ & 37 & $\begin{array}{l}\text { Preterm vaginal } \\
\text { delivery }\end{array}$ & $\begin{array}{l}2 \text { units random } \\
\text { donor platelets }\end{array}$ & Nil & Nil & $\begin{array}{l}\text { Macerated } \\
\text { girl baby, } \\
1.32 \mathrm{~kg}\end{array}$ \\
\hline Mrs B & $\begin{array}{l}\text { Moderate anaemia, } \\
\text { Preeclampsia,, } \\
\text { IUGR, Placenta } \\
\text { praevia, } \\
\text { Oligohydramnios, } \\
\text { IUD }\end{array}$ & & $29^{+2}$ & $\begin{array}{l}\text { Preterm vaginal } \\
\text { delivery }\end{array}$ & $\begin{array}{l}2 \text { units random } \\
\text { donor platelets } \\
\text { at Induction of } \\
\text { labour(IOL) }\end{array}$ & $\begin{array}{l}4 \text { units } \\
\text { random } \\
\text { donor } \\
\text { platelets on } \\
\text { PND1 }\end{array}$ & Nil & $\begin{array}{l}\text { Macerated } \\
\text { baby boy of } \\
470 \mathrm{~g}\end{array}$ \\
\hline Mrs B & IUGR, No anaemia & & $36^{+3}$ & $\begin{array}{l}\text { Full term vaginal } \\
\text { delivery }\end{array}$ & Nil & Nil & Nil & $\begin{array}{l}\text { Healthy, } \\
\text { boy, } 2.34 \\
\mathrm{~kg}\end{array}$ \\
\hline $\begin{array}{l}\text { Mrs } \\
M\end{array}$ & Moderate anaemia & & $37^{+5}$ & $\begin{array}{l}\text { Full term vaginal } \\
\text { delivery, Outlet } \\
\text { forceps for poor } \\
\text { maternal efforts }\end{array}$ & $\begin{array}{l}1 \text { unit } \\
\text { Pheresced } \\
\text { platelets at IOL } \\
\text { and } 1 \text { during } \\
\text { delivery }\end{array}$ & Nil & Nil & $\begin{array}{l}\text { Healthy, } \\
\text { girl, } 3.42 \\
\mathrm{~kg}\end{array}$ \\
\hline Mrs S & $\begin{array}{l}\text { Infection - Parotid } \\
\text { abscess at } 25 \text { weeks, } \\
\text { Oligohydramnios, } \\
\text { IUGR, IUD, No } \\
\text { anaemia }\end{array}$ & & $34^{+1}$ & $\begin{array}{l}\text { Preterm vaginal } \\
\text { delivery }\end{array}$ & Nil & Nil & Nil & $\begin{array}{l}\text { Macerated, } \\
\text { girl baby, } \\
391 \text { gm }\end{array}$ \\
\hline $\begin{array}{l}\text { Mrs } \\
\text { BR }\end{array}$ & $\begin{array}{l}\text { Moderate anaemia, } \\
\text { Blast crisis at } \\
\text { 24weeks, Preterm } \\
\text { labour }\end{array}$ & $\begin{array}{l}\text { Started on } \\
\text { Imatinib at } 24 \\
\text { weeks }\end{array}$ & $39^{+4}$ & $\begin{array}{l}\text { Elective LSCS at } \\
\text { maternal request }\end{array}$ & $\begin{array}{l}1 \text { unit Packed } \\
\text { cells preop \& } \\
1 \text { during } \\
\text { Caesarean } \\
\text { delivery } \\
\end{array}$ & $\begin{array}{l}1 \text { unit } \\
\text { Packed } \\
\text { cells on } \\
\text { POD4 }\end{array}$ & $\begin{array}{l}\text { Haematuria, } \\
\text { Epistaxis }\end{array}$ & $\begin{array}{l}\text { Healthy, } \\
\text { girl, } \\
2.3 \mathrm{~kg} \text {, Was } \\
\text { not } \\
\text { breastfed } \\
\end{array}$ \\
\hline $\begin{array}{l}\text { Mrs } \\
\text { MB }\end{array}$ & $\begin{array}{l}\text { Severe anaemia in } \\
\text { failure, Infection - } \\
\text { URTI, Preeclampsia, } \\
\text { HELLP, Previous } 2 \\
\text { LSCS }\end{array}$ & $\begin{array}{l}\text { 1unit Packed } \\
\text { cells at } 6^{\text {th }} \& \\
9^{\text {th }} \text { month and } \\
2 \text { units at } 8^{\text {th }} \\
\text { month } \\
\text { Iron sucrose } \\
\text { injections }\end{array}$ & $37^{+1}$ & $\begin{array}{l}\text { Elective LSCS - } \\
\text { Indication- } \\
\text { Previous } 2 \text { CS } \\
\text { and Preeclampsia }\end{array}$ & $\begin{array}{l}4 \text { units random } \\
\text { donor platelets } \\
\text { preop \& } 1 \text { unit } \\
\text { Packed cells \& } \\
\text { 1unit platelets } \\
\text { during } \\
\text { caesarean } \\
\text { delivery }\end{array}$ & $\begin{array}{l}\text { 1unit } \\
\text { Pheresced } \\
\text { platelets } \\
\text { given on } \\
\text { POD } 1 \& 6\end{array}$ & $\begin{array}{l}\text { Infection- } \\
\text { Pneumonia, } \\
\text { Fever, } \\
\text { Neutro- } \\
\text { penia, } \\
\text { ICU care }\end{array}$ & $\begin{array}{l}\text { Healthy, } \\
\text { girl, } \\
2.72 \mathrm{~kg}\end{array}$ \\
\hline Mrs S & $\begin{array}{l}\text { Severe anaemia } \\
\text { Chronic hypertension }\end{array}$ & $\begin{array}{l}1 \text { unit packed } \\
\text { cells at } 6^{\text {th }} \\
\text { month }\end{array}$ & 36 & $\begin{array}{l}\text { Emergency } \\
\text { LSCS for foetal } \\
\text { distress \& } \\
\text { worsening } \\
\text { maternal } \\
\text { condition } \\
\end{array}$ & $\begin{array}{l}8 \text { units random } \\
\text { donor platelets } \\
1 \text { unit Packed } \\
\text { cells } \\
4 \text { FFP }\end{array}$ & $\begin{array}{l}18 \text { random } \\
\text { donor } \\
\text { platelets } 3 \\
\text { units } \\
\text { Packed } \\
\text { cells } 4 \text { FFP }\end{array}$ & ICU care & $\begin{array}{l}\text { Healthy, } \\
\text { girl, } \\
1.23 \mathrm{~kg}\end{array}$ \\
\hline
\end{tabular}

LSCS - Lower segment caesarean section; POD - Post operative day; IOL - Induction of labour; UTI - Urinary tract infection; URTI - Upper respiratory tract infection; ICU - Intensive care unit; FFP - Fresh frozen plasma 
The New Indian Journal of OBGYN. 2021 (July-December);8(1)

\begin{tabular}{|c|c|c|c|c|c|c|c|c|c|c|c|c|c|}
\hline \multirow[t]{2}{*}{ Case } & \multicolumn{6}{|c|}{ During pregnancy } & \multicolumn{7}{|c|}{ After delivery, at discharge } \\
\hline & $\begin{array}{l}\text { Hb } \\
\text { g/dL }\end{array}$ & $\begin{array}{l}\text { TC } \\
\times 10^{9} / L\end{array}$ & $\begin{array}{l}\text { Neutrophil } \\
\text { Count } \\
\left(\times 10^{9} / \mathrm{L}\right)\end{array}$ & $\begin{array}{l}\begin{array}{l}\text { Platelet } \\
\text { count } \\
\left(\times 10^{9} / \mathrm{L}\right)\end{array} \\
\end{array}$ & PT/INR & APTT & $\begin{array}{l}\text { Retic } \\
\text { count } \\
\% \\
\end{array}$ & $\begin{array}{l}\text { Hb } \\
\text { g/dL }\end{array}$ & $\begin{array}{l}\text { TC } \\
\mathbf{x 1 0 ^ { 9 } / L}\end{array}$ & $\begin{array}{l}\text { Neutrophil } \\
\text { Count } \\
\text { (x 10 } 1 \text { L) }\end{array}$ & $\begin{array}{l}\begin{array}{l}\text { Platelet } \\
\text { count } \\
\left(\times 10^{9} / L\right)\end{array} \\
\end{array}$ & PT/INR & $\begin{array}{l}\text { AP } \\
\text { TT }\end{array}$ \\
\hline $\begin{array}{l}\text { Mrs } \\
\text { M }\end{array}$ & 7.2 & 0.68 & O.0068 & 84 & $10.8 / 0.8$ & 22.1 & 0.62 & 4.6 & 0.82 & 0.0082 & 80 & * & $*$ \\
\hline $\begin{array}{l}\text { Mrs } \\
\text { M }\end{array}$ & 9.2 & 1.113 & 0.169 & 55 & $9 / 0.8$ & 24.4 & $*$ & 12 & 2.18 & 0.436 & 120 & $11.6 / 0.8$ & 33 \\
\hline $\begin{array}{l}\text { Mrs } \\
\text { S }\end{array}$ & 6.7 & 4.78 & 2.581 & 61 & $9.8 / 0.8$ & 25 & $*$ & 9.7 & 7.57 & 6.131 & 63 & $\begin{array}{l}11.8 / \\
1.01\end{array}$ & 24.6 \\
\hline $\begin{array}{l}\text { Mrs } \\
\text { B } \\
\end{array}$ & 9.1 & 3.84 & 1.420 & 76 & $10.6 / 0.9$ & 29.4 & $*$ & 9.7 & 6.14 & 2.578 & 39 & $*$ & $*$ \\
\hline $\begin{array}{l}\text { Mrs } \\
\text { B } \\
\end{array}$ & 11.2 & 5.54 & 3.434 & 199 & $10.4 / 0.8$ & 28 & $*$ & 12.1 & 7.99 & 4.394 & 136 & * & * \\
\hline $\begin{array}{l}\text { Mrs } \\
M\end{array}$ & 8.8 & 4.73 & 2.696 & 30 & $9.6 / 0.8$ & 26 & 1.75 & 8.4 & 8.93 & 6.697 & 139 & $*$ & $*$ \\
\hline $\begin{array}{l}\text { Mrs } \\
\text { S }\end{array}$ & 11.3 & 97.83 & 55.763 & 378 & $\begin{array}{l}11.8 / \\
1.03 \\
\end{array}$ & 30 & 2.9 & 12.8 & 8.80 & 5.258 & 296 & $\begin{array}{l}11.8 / \\
1.08 \\
\end{array}$ & 28.8 \\
\hline $\begin{array}{l}\text { Mrs } \\
\text { BR }\end{array}$ & 8.3 & 6.45 & 3.870 & 311 & $10.5 / 0.9$ & 26.8 & 0.39 & 9.8 & 7.10 & 4.615 & 242 & $10.5 / 0.9$ & 22.9 \\
\hline $\begin{array}{l}\text { Mrs } \\
\text { MB } \\
\end{array}$ & 6.6 & 15.50 & 1.023 & 40 & $10.9 / 0.9$ & 26.8 & 1.26 & 9.3 & 23.60 & 18.644 & 45 & $10.9 / 0.9$ & 21.2 \\
\hline $\begin{array}{l}\text { Mrs } \\
\mathrm{S} \\
\end{array}$ & 6.9 & 7.40 & 6.142 & 65 & $9.9 / 0.9$ & 25.6 & * & 10.6 & 7.50 & 5.850 & 24 & $10.9 / 0.9$ & 25.1 \\
\hline
\end{tabular}

\section{Discussion}

The prevalence of bone marrow disorders complicating pregnancy in our study was $0.08 \%$. There are very few studies in literature stating the prevalence, thus showing its rarity.

The prevalence of aplastic anaemia as per literature is 2-6 /million with 2 to 3fold higher incidence in Asia than in Europe ${ }^{6,7,12}$. We encountered 6 cases among 12420 pregnancies $(0.04 \%)$ over a period of 5 years. This could be due to referral to a tertiary care center. Also, the increase in prevalence of acquired causes of AA along with availability of better treatment options like immunosuppressive drugs, anti thymocyte globulin, bone marrow transplantation and haemopoetic growth factors, more women are in remission and conceiving as fertility is unaffected ${ }^{11}$. This could also explain the presence of younger mothers found in our cohort.

The incidence of leukemia during pregnancy is reported to be 1 in 75,000-100,000 pregnancies. AML accounts for two-thirds of all acute leukemia cases reported during pregnancy ${ }^{13-15}$. Chronic myeloid leukaemia constitutes $10 \%$ of pregnancy associated leukaemias ${ }^{16}$. Although the reported incidence of MDS is approximately $10-12$ patients in 100 000 people, recent studies have indicated that the incidence rate has increased in young patients ${ }^{17}$. We had 2 cases of CML, 1 of AML and 1 of MDS.

Maternal and fetal complications frequently occur in patients with severe AA ${ }^{11}$. We had 2 cases of very severe AA - both had antenatal complications, but they did not receive antenatal transfusions or drugs for the same and had good neonatal outcomes.

Preexisting aplastic anaemia associated with pregnancy has a better prognosis compared to when it occurs during pregnancy ${ }^{2,8}$. We too noted better maternal and foetal outcomes as $83 \%(5 / 6)$ were diagnosed to have AA prior to pregnancy. Women whose disease is in remission before conception and those with normal platelet counts are more likely to have an uneventful pregnancy ${ }^{18}$. 4/6 women were in remission, of which two had uneventful pregnancies despite three of them having thrombocytopenia. 1 woman with CML was in remission. Pregnancy should be planned during chronic phase, while in remission for more than 2 years ${ }^{16,19}$ as was seen in our case too.

Normal blood counts before conception do not guarantee that relapse of AA will not occur ${ }^{11,18}$. The blood cell levels may unpredictably decrease during pregnancy because of hormonal changes, altered immunity during pregnancy and a 
decreased marrow reserve further drawn by the demands of pregnancy ${ }^{11,18}$. Tichelli et al have reported a relapse rate of $19 \%$, more often in the second and third trimester ${ }^{18}$. Only 1 woman with AA had a relapse at 36 weeks, developed $\mathrm{PNH}$, received multiple transfusions and had an IUD at term in our study. The women with AML and MDS had relapses requiring frequent transfusion of blood products in the antepartum and peripartum period. Both had a stormy postoperative period requiring ICU care.

There is concern regarding teratogenicity of the drugs used during pregnancy. Androgen is an alternative to immunosuppressive therapy; it improves the platelet count in $37 \%$ of cases. However, its use during pregnancy exposes to the risk of virilization of the female fetus ${ }^{20}$. The foetus of the woman on danazol had a congenital anomaly whereas the neonates of those who received steroids and cyclosporine were normal in our study. The efficacy of corticosteroids is also equivocal ${ }^{4}$. Cyclosporine $(300 \mathrm{mg} /$ day) has been used in severe aplastic anemia after 20 weeks of pregnancy though data regarding their use in pregnancy with aplastic anemia is limited. However, experience from pregnancy following organ transplant shows that cyclosporine is apparently not teratogenic ${ }^{3,4,18}$. Though it is excreted in milk, fetal growth and development were found to be normal $^{4}$.

The advent of tyrosine kinase inhibitors (TKIs) like Imatinib has revolutionized treatment of CML, improving prognosis and thereby its survival to as much as $85 \%{ }^{19}$. Imatinib, a category D drug, has become the mainstay of treatment $16,21,22$. In addition to the risk of spontaneous abortion, miscarriage, stillbirth, preterm delivery and foetal growth restriction, its teratogenic effects include exomphalos, omphaloceles, pulmonary hypoplasia, duplex kidneys, renal agenesis, skeletal malformations (craniosynostosis, shoulder anomaly, and scoliosis) ${ }^{16,22}$. As Imatinib is secreted in breast milk ${ }^{16}$, breast feeding must be discouraged $^{22,23}$.

Similarly, in our study, among the 2 women with CML, 1 was diagnosed in pregnancy and received Imatinib from 7 weeks due to severe disease and had optimal response as evidenced by BCR ABL RT PCR at 3 and 6 months of $30 \%$ and $0.8 \%$; but the foetus developed IUGR and she had an IUD at 34 weeks. The other patient was in remission and Imatinib stopped prior to conception; she was restarted on Imatinib due to blast crisis at 24 weeks. She delivered a healthy baby at term with no congenital anomalies and chose not to breast feed her baby. Most authors recommend that
Imatinib therapy should be interrupted 3 months prior to conception and for the duration of pregnancy and restarted soon after delivery ${ }^{16,19}$.

We observed that all our women with AA had atleast one obstetrics complication and $33 \%$ had neonatal complications in concurrence with Perez et al ${ }^{5}$. We had a lower mean gestational age at delivery as leucopenia leads to sepsis, chorioamnionitis and preterm labour ${ }^{5}$. Due to immune mediated mechanism, hypertension, oligohydramnios, IUGR and IUD are common ${ }^{5}$.

As per literature, the rate of preterm labour was $12 \%$, IUD $16.7 \%$, stillbirth $15 \%$, spontaneous miscarriage $16.7 \%$, preeclampsia 5.9\%, APH $15.7 \%$ and small gestational age $11.8 \%$ with AA ${ }^{4,8,24}$. We noticed a higher rate of complications (preterm labour 50\%, preeclampsia 33\%, IUGR and IUD 33\%) compared to other authors because most of our patients were unbooked with poor compliance to treatment or follow up. Our women were younger (26 vs 31 yrs), delivered preterm (35 vs 34 weeks) and had LBW babies ( 2 vs $2.5 \mathrm{~kg}$ ) compared to Chen et al ${ }^{8}$. Despite this $66 \%$ had good neonatal outcomes in our study.

CML is not associated with an increase in premature infants, low birth weight, or abortion rates, which were concerns previously ${ }^{16}$. Abortions, preterm deliveries, IUGR and stillbirths are common with AML ${ }^{14}$. The degree of anaemia during pregnancy and gestational hypertension were significantly associated with poor pregnancy outcome in women with MDS ${ }^{17}$. The greater the intensity of cytopenias, the more frequent the adverse outcomes ${ }^{17}$. Both the patients with AML and MDS had hypertension and severe anaemia in our cohort.

Majority of the women with AA (66\%) had vaginal deliveries and caesarean sections were done for obstetrical indications in our study. Our data concurs with other authors, who have said that vaginal deliveries are preferred in women with $\mathrm{AA}^{4,5,11}$. However, the incidence of caesarean section is high, especially among patients with severe AA due to increased frequency of antenatal complications ${ }^{11,18}$. The women with AML, CML and MDS too had caesarean deliveries for obstetrics indications in our study. Fracchiolla et al have advocated caesarean delivery near term $(>35-37$ weeks) to minimize risks for mother and foetus with AML ${ }^{13}$.

The incidence of hemorrhagic complications during the peripartum period requiring blood transfusions, in the presence of thrombocytopenia in AA has been reported to be as high as $75 \%{ }^{5}$. None of the women in our study had PPH despite $90 \%$ of them having thrombocytopenia because of 
The New Indian Journal of OBGYN. 2021 (July-December);8(1)

prophylactic transfusions. $70 \%$ received supportive therapy in the form of repeated blood and platelet transfusions to keep $\mathrm{Hb}$ at $10.5 \mathrm{~g} / \mathrm{dl}$ and platelet count at $20 \times 10^{9} / \mathrm{L}$ as is recommended by several authors ${ }^{4,5,12}$. In poor resource settings where facilities for administration of immunesuppressive therapy or bone marrow transplantation are not available, pregnancies can be successfully managed by supportive therapy ${ }^{11}$.

Fever and sepsis are common in puerperium due to neutropenia ${ }^{5,11}$. In our study only $30 \%$ developed fever or sepsis due to the strict aseptic practices followed in the care of these patients. ICU backup is crucial to reduce maternal and perinatal morbidity and mortality, as was seen in our cohort where $30 \%$ of the mothers and $40 \%$ of the neonates required ICU care.

Blood counts return to pre pregnant levels in 1 to 6 months after delivery ${ }^{18}$. Our cohort also showed an improvement of blood counts after delivery either due to transfusion of blood products peripartum or due to withdrawal of hormones which suppress haematopoiesis during pregnancy ${ }^{2,3,5}$.

The mortality in aplastic anaemia associated with pregnancy is reported at $20 \%-60 \%$ and is mainly due to haemorrhage and sepsis ${ }^{3,8}$. Currently maternal mortality is put at $2.7 \%$ due to improvement in supportive care ${ }^{11}$. There was no maternal mortality in our cohort.

The retrospective nature of the study and loss of maternal and neonatal follow up are some of the limitations of this study. Preterm labour, preeclampsia, IUGR, IUD, APH, $\mathrm{PPH}$, sepsis and anaemia are common antenatal complications encountered in pregnancy with bone marrow disorders. Despite this, most of our women had good maternal and foetal outcomes. There was no PPH or maternal mortality in our cohort.

\section{Conclusion}

Multidisciplinary approach, preconceptional counseling, good antenatal care, frequent monitoring of blood counts, delivery at a tertiary care centre, prompt treatment of infections and sepsis, prophylactic transfusions to keep $\mathrm{Hb}$ at $10 \mathrm{~g} / \mathrm{dl}$ and platelet count at $50 \times 10^{9} / \mathrm{L}$ for caesarean delivery and $20 \times 10^{9} / \mathrm{L}$ for vaginal delivery, optimal use of chemotherapeutic drugs can ensure a successful obstetrical and neonatal outcome for pregnancies complicated by bone marrow disorders.

\section{Conflict of interest: None. Disclaimer: Nil.}

\section{References}

1. Neal SY. Bone marrow failure syndrome including Aplastic Anaemia and Myelodysplasia In: Fauci AS, Kasper DL, Hauser SL, Longo DL, Jameson JL, Loscalzo J, editors. Harrison's Principles of Internal Medicine. 20th ed. New York: McGraw-Hill Education; 2018 , p. 723.

2. Deka D, Banerjee N, Roy KK, Choudhary VP, Kashyap R, Takkar D. Aplastic anaemia during pregnancy: variable clinical course and outcome. Eur J Obstet Gynecol Reprod Biol. 2001; 94(1):152-4.

3. Deka D, Malhotra N, Sinha A, Banerjee N, Kashyap R, Roy KK. Pregnancy associated aplastic anemia: maternal and fetal outcome. J Obstet Gynaecol Res. 2003; 29: 67-72.

4. Rathore S, Pramanick A, Regi A, Lionel J. Aplastic anemia in pregnancy. J Obstet Gynaecol India. 2014; 64(1): 26-8.

5. Riveros-Perez E, Hermesch AC, Barbour LA, Hawkins JL. Aplastic anemia during pregnancy: a review of obstetric and anesthetic considerations. Int J Womens Health. 2018;10:117-25.

6. John CO, Korubo K, Ogu R, Mmom CF, Mba AG, Chidiadi EA, et al. Management of aplastic anaemia in pregnancy in a resource poor centre. Pan Afr Med J. 2016; 24: 277.

7. 7. Mishra V, Roy P, Vyas B, Aggarwal R, Choudhary S, Mehta N. Aplastic anemia in pregnancy: a rare case report. Int J Reprod Contracept Obstet Gynecol. 2016; 5: 4506-8.

8. Chen KJ, Chang YL, Chang H, Su SY, Peng HH, Chang $\mathrm{SD}$, et al. Long-term outcome of pregnancy complicating with severe aplastic anemia under supportive care. Taiwan J Obstet Gynecol. 2017; 56(5): 632-5.

9. Good Clinical Practice Recommendations for Iron Deficiency Anemia in Pregnancy (IDA) in Pregnancy in India. J Obstet Gynaecol India. 2011; 61(5): 569-71.

10. 10. Camitta BM, Storb R, Thomas ED. Aplastic anemia. Parts I and II. Pathogenesis, diagnosis, treatment, and prognosis. N Engl J Med. 1982; 306: 645-52.

11. Pajor A, Janossa M, Kelemen E. Pregnancy in aplastic anemia treated with fetal liver and bone marrow hemopoietic cells and antithymocyte globulin. Arch Gynecol Obstet 1992; 251(4): 207-10. 
The New Indian Journal of OBGYN. 2021 (July-December);8(1)

12. Stibbe KJ, Wildschut HI, Lugtenburg PJ. Management of aplastic anemia in a woman during pregnancy: a case report. J Med Case Rep. 2011; 5: 66.

13. Fracchiolla NS, Sciumè M, Dambrosi F, Guidotti F, Ossola MW, Chidini G, et al. Acute myeloid leukemia and pregnancy: clinical experience from a single center and a review of the literature. BMC Cancer. 2017; 17(1):442.

14. Alrajhi AM, Alhazzani SA, Alajaji NM, Alnajjar FH, Alshehry NF, Tailor IK. The use of 5-azacytidine in pregnant patient with Acute Myeloid Leukemia (AML): a case report. BMC Pregnancy Childbirth. 2019; 19(1):1-4.

15. Seegars MB, Powell BL, Howard DS. Acute Myeloid Leukemia in Pregnancy. Blood. 2017;130(1):5035.

16. Madabhavi I, Sarkar M, Modi M, Kadakol N. Pregnancy Outcomes in Chronic Myeloid Leukemia: A Single Center Experience. J Glob Oncol. 2019;5:1-11.

17. Yang Z, Mei-Ying L, Shan-Mi W, Xiao-Hui Z. Pregnancy and myelodysplastic syndrome: an analysis of the clinical characteristics, maternal and fetal outcomes. J Matern Fetal Neonatal Med. 2015; 28(18): 2155-9.

18. Tichelli A, Socié G, Marsh J, Barge R, Frickhofen N, McCann S, et al. Outcome of Pregnancy and Disease Course among Women with Aplastic Anemia Treated with Immunosuppression. Ann Intern Med. 2002; 137:164 -72.

19. Mikhael S, Pascoe A, Prezzato J. Recurrence of Chronic Myeloid Leukemia during Pregnancy Subsequently
Achieving Complete Medical Remission. Case Rep Oncol Med. 2017; 2017: 5651064.

20. Amine B, Najat L, Rajaa T. Severe aplastic anemia and pregnancy: case report. Int J Fam Commun Med. 2019; 3(3):116-7.

21. Yadav U, Solanki SL, Yadav R. Chronic myeloid leukemia with pregnancy: successful management of pregnancy and delivery with hydroxyurea and imatinib continued till delivery. J Cancer Res Ther. 2013; 9(3): 484-6.

22. Bhandari A, Rolen K, Shah BK. Management of chronic myelogenous leukemia in pregnancy. Anticancer Res. 2015; 35(1):1-11.

23. Moura AC, Delamain MT, Duarte GBO, Lorand-Metze I, Souza CA, Pagnano KBB. Management of chronic myeloid leukemia during pregnancy: a retrospective analysis at a single center. Hematol Transfus Cell Ther. 2019; 41(2):125-8.

24. Idler J, Patwardhan S, Gonik B. Pregnancy After Bone Marrow Transplantation for Aplastic Anemia [37Q]. Obstetrics \& Gynecology. 2018;131:194S.

\section{Rao Preethi Venkatachala ${ }^{1}$, C N Sheela ${ }^{2}$}

${ }^{1}$ Assistant Professor, Department of Obstetrics and Gynaecology, St Johns Medical College and Hospital, Bengaluru, Karnataka, India; ${ }^{2}$ Professor, Department of Obstetrics and Gynaecology, St Johns Medical College and Hospital, Bengaluru, Karnataka, India. 